Article

\title{
Analysis of protein intramolecular and solvent bonding on example of major synovial fluid component
}

\author{
Krzysztof Domino ${ }^{1}$, Damian Bełdowski ${ }^{2}$, Adam Mazurkiewicz ${ }^{3 *}$, Janusz \\ Musiał ${ }^{3}$, Małgorzata Słomion ${ }^{4}$ and Robert Dobosz ${ }^{5}$ \\ 1 Institute of Theoretical and Applied Informatics, Polish Academy of Sciences kdomino@iitis.pl \\ 2 Institute of Mathematics, Jagiellonian University; damian.beldowski@student.uj.edu.pl \\ 3 Mechanical Engineering Department, UTP University of Science and Technology Kaliskiego 7 Street 85-789 \\ Bydgoszcz, Poland, adam.mazurkiewicz@utp.edu.pl (A.M), janusz.musial@utp.edu.pl (J.M.) \\ 4 Faculty of Management, UTP University of Science and Technology, Kaliskiego 7 Street, 85-789 Bydgoszcz \\ Poland, malgorzata.slomion@utp.edu.pl \\ 5 Institute of Mathematics and Physics, UTP University of Science and Technology, Kaliskiego 7 Street, 85- \\ 796 Bydgoszcz, Poland, robertd@utp.edu.pl \\ * Correspondence: adam.mazurkiewicz@utp.edu.pl; Tel.: +48-52-340-82-29
}

\begin{abstract}
:
In this paper we review dynamics and roughness of bonds in proteins on example of albumin, that is important from the physiological point of view. We have performed computer simulations of albumin chain. Statistics were collected by performing many simulations realizations for each experimental setting. We concentrate on hydrogen bonds, cation- $\pi$ and $\pi-\pi$ interactions and NP contacts. Histograms of hydrogen bonds length are positively skewed in contrary to histograms of interactions and HP contacts that are negatively skewed. Scaling exponents of power spectra of energies of bonds / interactions /contacts are in range -0.2 to -0.5 and significantly differ between various hydrogen bonds or interactions. Varying scaling of such spectra can be used to classify between distinct bonds or contacts. Concerning particular amino-acids, largest amount of $\mathrm{HBO} \mathrm{H}_{2} 0$ bonds are between Glutamate (GLU) amino-acids and water particle, while large amount of $\mathrm{HBO}$ bonds are formed with Lysine (LYS). For HP contacts the mayor role plays Phenylalanine (PHE) and Leucine (LEU) amino-acids. From decay curves HBO $\mathrm{H}_{2} \mathrm{O}$ bonds decays in fastest rate, while $\mathrm{HBO}$ bonds and $\mathrm{HP}$ contacts at slowest rate. We present as well decay curves of bonds formed by particular amino-acids, that gives interesting results.
\end{abstract}

Keywords: human albumin; hydrogen bonds; HP contacts; $\pi-\pi$ / cation- $\pi$ interactions; bonds roughness; decay curve; power spectrum; interaction between amino-acids; 


\section{Introduction}

Human Serum Albumin (HSA) is an abundant multifunctional non-glycosylated, negative charged plasma protein [1]. It is also the main component of the synovial fluid [2]. Due to the high concentration in the circulatory system $(\sim 0.6 \mathrm{mM}$ in plasma) and from its ability to bind an extraordinarily diverse range of drugs, metabolites, and fatty acids, it shows importance in the functioning of organisms [3]. Human albumin constitutes some $50 \%$ of the protein present in the plasma of normal healthy individuals [4]. It is relatively small to other plasma proteins $(66 \mathrm{kd})$, composed of 585 amino acids with few tryptophan or methionine residues but an abundance of charged residues such as lysine and aspartic acids and no prosthetic groups or carbohydrate [5]. The structure of the HSA is shown in Fig 1.

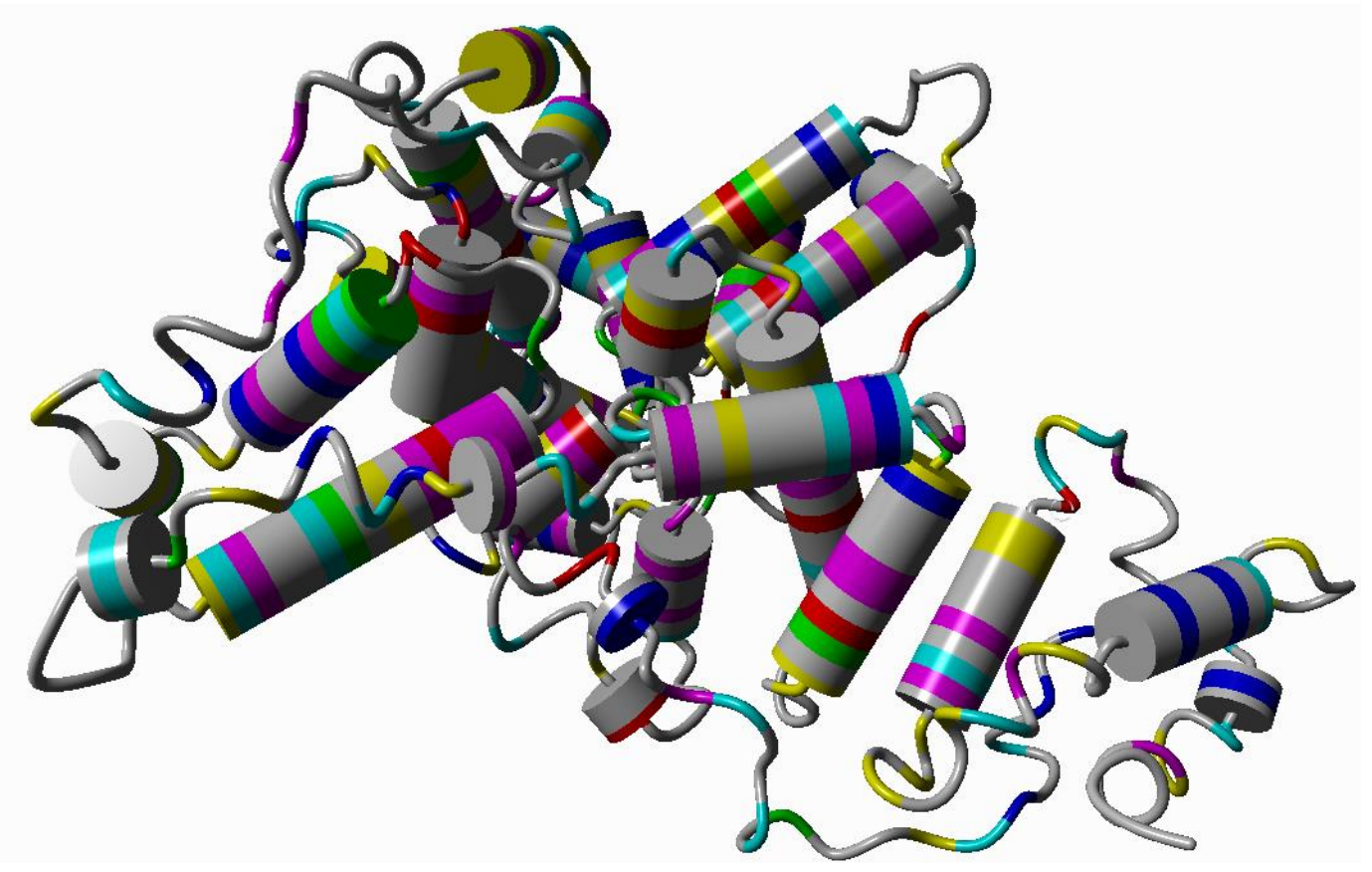

Figure 1. Visualization of albumin molecule in a cartoon form. Several amino acid has been colored to show their position in the molecule. Colors read as follows: pink-LEU, yellow-LYS, green-TYR, cyan-GLU, blue-PHE, red-ARG. Tubes reflects alpha helices.

HSA, like any other polymer, consists of monomers, in this case amino acids. They are combined in a specific sequence and interact with each other. To understand the structure of albumin (and other proteins) we need to know what influence non-covalence interactions such as hydrogen bond (HB), hydrophobic contacts (HP), $\pi-\pi$ and cation- $\pi$ interactions have on the formation of the protein chain. An important aspect is also which amino acids most often interact with each other and what types of interactions they are [6-10]. Thus, these interactions are studied in the paper.

The hydrogen bond is an attractive interaction between a hydrogen atom from a molecule or a molecular fragment $\mathrm{X}-\mathrm{H}$ in which $\mathrm{X}$ is more electronegative than $\mathrm{H}$, and an atom or a group of atoms in the same or a different molecule $Y$, in which 
there is evidence of bond formation X-H..Y [11]. As we can see in Table 1 [12], the strength of hydrogen bonds depends on the geometry of molecules.

Table 1. Characteristic of the three types of hydrogen bonds.

\begin{tabular}{cccc}
\hline Parameters & Strong HB & Medium HB & Weak HB \\
\hline Interaction type & Strongly & Mostly & Electrostatic/dispersed \\
& covalent & electrostatic & \\
$\mathrm{H} \cdots \mathrm{Y}[\AA]$ & $1.2-1.5$ & $1.5-2.2$ & $>2.2$ \\
$\mathrm{X}-\mathrm{H}[\AA]$ & $0.08-0.25$ & $0.02-0.08$ & $<0.02$ \\
$\mathrm{X}-\mathrm{H}$ Vs. H $\cdots \mathrm{Y}$ & $\mathrm{X}-\mathrm{H} \approx \mathrm{H} \cdots \mathrm{Y}$ & $\mathrm{X}-\mathrm{H}<\mathrm{H} \cdots \mathrm{Y}$ & $\mathrm{X}-\mathrm{H}<<\mathrm{H} \cdots \mathrm{Y}$ \\
$\mathrm{X} \cdots \mathrm{Y}[\AA]$ & $2.2-2.5$ & $2.5-3.2$ & $>3.2$ \\
$\mathrm{X}-\mathrm{H} \cdots \mathrm{Y}\left[{ }^{\circ}\right]$ & $170-180$ & $>130$ & $>90$ \\
$\mathrm{H}-$ bond energy & $15-40$ & $4-15$ & $<4$ \\
{$[\mathrm{kcal} / \mathrm{mol}]$} & & & \\
\hline
\end{tabular}

Figure 2 shows a fragment of the HSA molecule with marked hydrogen bonds. As we can see, they are an important aspect determining the structure of albumin.

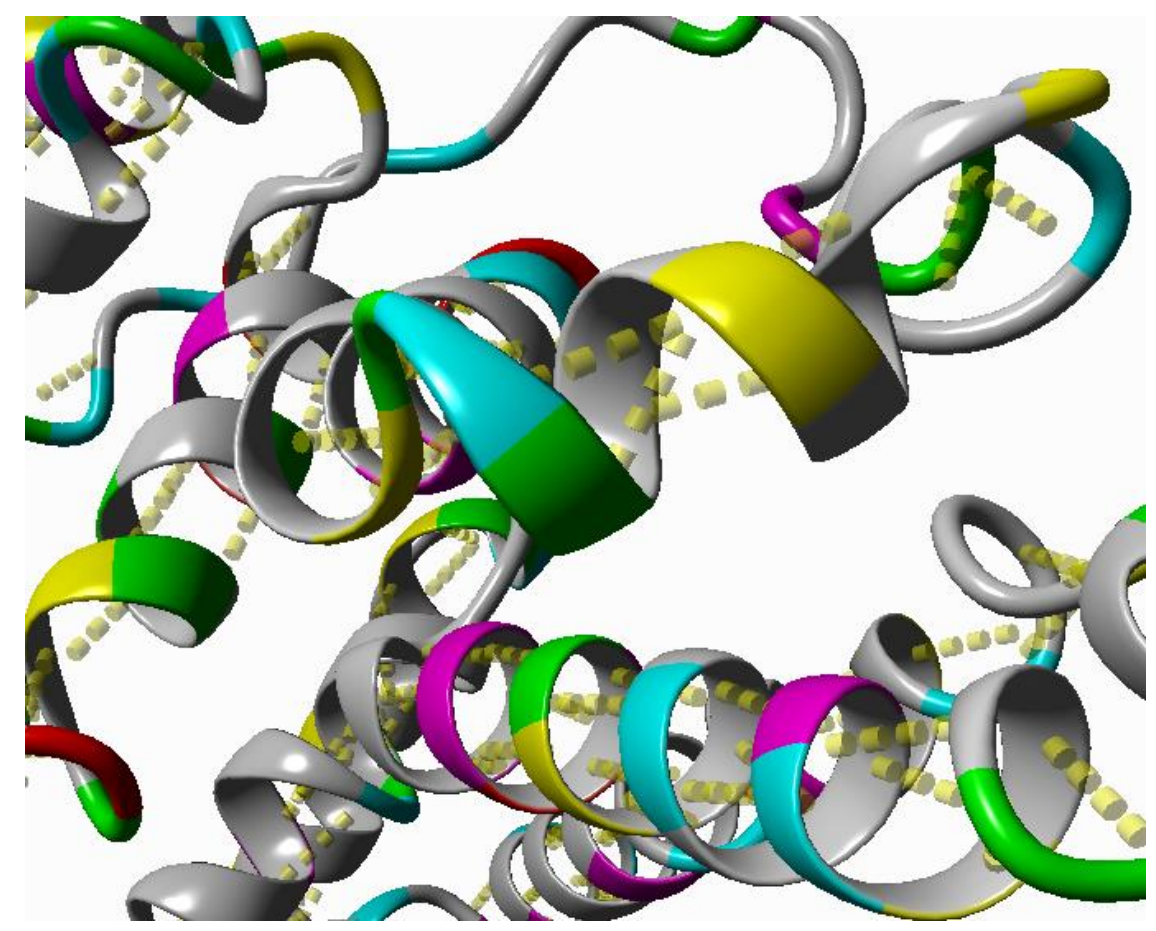

Figure 2. Visualization of albumin HB. Several amino acid has been colored to show their position in the molecule. Colors read as follows: pink-LEU, yellow-LYS, green-ALA, cyan-GLU, red-ARG.

In organic aromatic systems containing delocalized $\pi$-electrons (such as that of benzene, tryptophan, phenylalanine or tyrosine) there are non-covalent interactions such as cation $-\pi$ interaction and $\pi-\pi$ stacking (Fig.3). These are weak interactions between the delocalized $\pi$ electrons of one aromatic ring and $\pi$ electrons of the other ring (repulsive) or positively charged moieties or cation (attracting) [6]. 

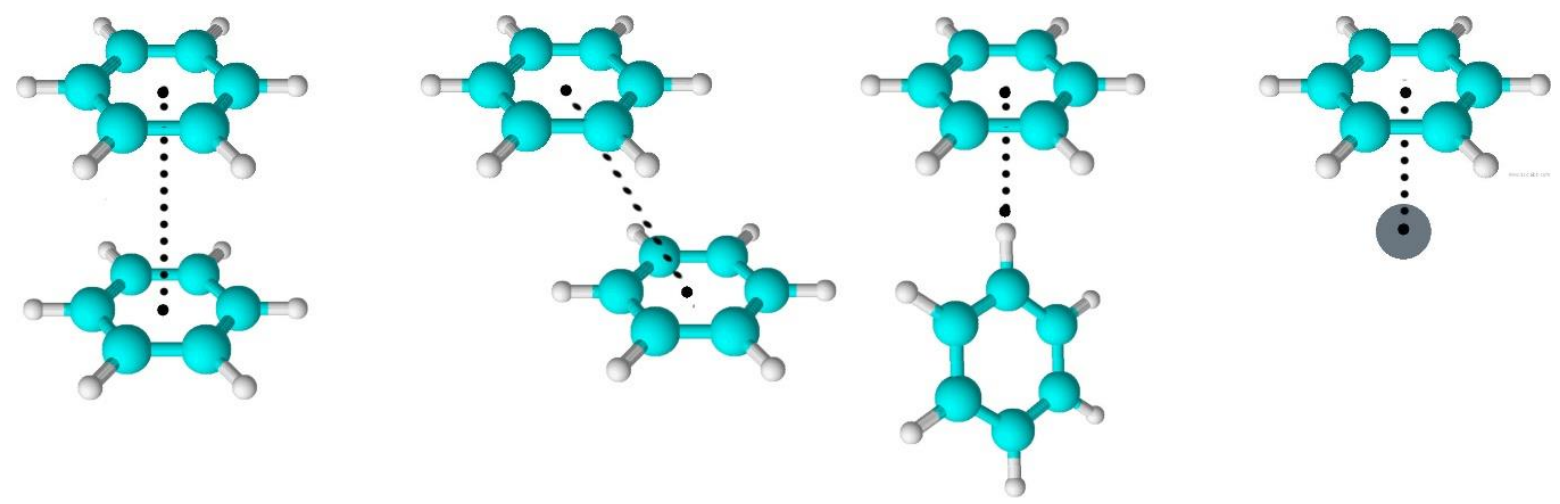

Figure 3. Examples of $\pi-\pi$ interactions (3 panels from the left: sandwich, parallel-displaced, T-shape, respectively) and cation $-\pi$ interactions (on the right).

While considering protein dynamics it is important to mention that amino acids containing hydrophobic groups in the aquatic environment begin to interact with each other (form aggregates). These interactions lead to the minimalization of the surface area of protein available to water and are called the 'hydrophobic effect' [1314]. Hence such dynamic is complex and interesting to examine.

The aim of this study is to present a statistical analysis of basic interaction occurring in albumin containing aqueous solution. Namely, hydrogen bonding between solute and solvent were examined as well as intramolecular solute bonding. Moreover, three other major interactions were analyzed: hydrophobic effect, hydrogen bond, $\pi-\pi$ and cation $-\pi$.

\section{Materials and Methods}

Simulation box containing albumin (downloaded from the protein data bank https://www.rcsb.org/structure/1E78) was filled with water (TIP3P model) containing $0.9 \% \mathrm{NaCl}$ (physiological solution) in the amount corresponding to established water density. System minimalization was performed with a time step 1 fs for 1000 steps. For the presented configuration, the volume box is about $864 \mathrm{~nm}^{3}$ $(11.2 \mathrm{~nm} \times 8.3 \mathrm{~nm} \times 9.3 \mathrm{~nm})$, with a periodic bondary condition applied. All-atom isothermal-isobaric (NPT) ensemble simulations were performed under the following conditions: temperature 300:3:315K, with a time step of 2 fs. Berendsen barostat and thermostat with a relaxation time of $1 \mathrm{fs}$ were used to maintain constant temperature and pressure. The simulation results were sampled every 50ps, whereas the simulation lasted 100 ns. A total of 2000 data points were collected to analyze. An Assisted Model Building with Energy Refinement (AMBER) force field has been employed to mimic the interactions between molecules. The functional form of the AMBER03 force field is:

$$
E_{\text {total }}=E_{b o n d s}+E_{\text {angles }}+E_{\text {dihedrals }}+E_{v d W}+\text { Eelectrostatic }
$$

where Ebonds represents the energy between covalently bonded atoms, Eangles represents the energy due to the geometry of electron orbitals involved in covalent 
bonding, Edihedrals represents the energy for twisting a bond due to bond order (e.g., double bonds) and neighboring bonds or lone pairs of electrons, Evdw represents the non-bonded energy between all atom pairs (van der Waals energy), and Eelectrostatic represents the electrostatic interactions between all atom pairs. Further, deploying the YASARA Structure package, molecular dynamics simulations were performed to study PL-HA interactions. The methods for surface area calculation follow that previously utilized $[15,16]$.

Hydrogen bond (HB) definition is defined by YASARA as follows. Hydrogen bond energy is strictly not less than $6.25 \mathrm{~kJ} / \mathrm{mol}$ (or $1.5 \mathrm{kcal} / \mathrm{mol}$ ) (weak and very weak bonds are omitted). This number is only $25 \%$ of the optimal $25 \mathrm{~kJ} / \mathrm{mol}$ energy value. Unit of the next formula is express in $\mathrm{kJ} / \mathrm{mol}$ and is depended of two scaling factors and the Hydrogen-Acceptor distance:

$$
E_{H B}=25 \cdot \frac{\chi^{(D-A-H)} \cdot \chi^{(H-A-X)} \cdot\left[2.6-\max \left(\operatorname{dis}_{(H-A)}, 2.1\right)\right]}{0.5}
$$

where in the first from above scaling factors depends from the angle formed by Donor-Hydrogen-Acceptor, and the second scaling factor is obtained from the angle formed by Hydrogen-Acceptor- $X$. The latter $X$ stands for the atom covalently bond to the acceptor. The first and the second scaling factor can take values from the range $[0,1]$ (angles in both formulas are expressed in radians angles)

$$
\chi^{(D-A-H)}=\left\{\begin{array}{lll}
0 & \text { if angle is beteween } & {\left[0, \frac{5 \pi}{9}\right) \text { radians, }} \\
x \in[0,1] & \text { if angle is beteween } & {\left[\frac{5}{9} \pi, \frac{11 \pi}{12}\right] \text { radians, }} \\
1 & \text { if angle is beteween } \quad\left(\frac{11 \pi}{12}, \pi\right] \text { radians, }
\end{array}\right.
$$

in the case when $X$ is a heavy atom, then the second scaling factor attains following values

$$
\chi^{(H-A-X)}=\left\{\begin{array}{lll}
0 & \text { if angle is beteween } & {\left[0, \frac{17 \pi}{36}\right) \text { radians, }} \\
x \in[0,1] & \text { if angle is beteween } & {\left[\frac{17}{36} \pi, \frac{19 \pi}{36}\right] \text { radians, }} \\
1 & \text { if angle is beteween } & \left(\frac{19 \pi}{36}, \pi\right] \text { radians, }
\end{array}\right.
$$

in the case when $\mathrm{X}$ is a hydrogen atom, then smaller angles are allowed. In such scenario the scaling factor has following form: 


$$
\chi^{(H-A-X)}=\left\{\begin{array}{lll}
0 & \text { if angle is beteween } & {\left[0, \frac{5 \pi}{12}\right) \quad \text { radians, }} \\
x \in[0,1] & \text { if angle is beteween } \quad\left[\frac{5}{12} \pi, \frac{17 \pi}{36}\right] \quad \text { radians, } \\
1 & \text { if angle is beteween } \quad\left(\frac{17 \pi}{36}, \pi\right] \text { radians, }
\end{array}\right.
$$

For the case when the Acceptor forms has more than one covalent bond, then the one witch has the lowest scaling factor is taken into calculation.

The hydrophobic interaction strength between hydrophobic atoms (carbon based) is calculated as follows. Hydrophobic atoms are identified and assigned an atom type from the groups depicted as type 1 for carbon atoms with three or more bond hydrogen atoms $\left(-\mathrm{CH}_{3}\right)$, type 2 for carbon atoms with two hydrogen atoms or one hydrogen atom plus three carbon atoms bond $\left(-\mathrm{CH}_{2}-, \mathrm{HCC}_{3}\right)$, and type 3 for aromatic ring carbon atoms with only carbon and hydrogen atoms bond [9].

YASARA provided $\pi-\pi$ command. Those $\pi-\pi$ methods are related to interactions which are non-covalent interactions involving electrons in pi bonds. Due to the fact in this case non-covalent interaction are taken to account so there is possibility to separate them into Van der Waals attraction (always present) and electrostatic interactions.

The term ' $\pi-\pi$ interaction' is somewhat misleading, because it creates the impression that only $\pi$ electrons are required (leading to misconceptions like a sandwich of two Phe side-chains being a favorable $\pi-\pi$ interaction, which is not the case).

However, just like hydrogen bond acceptors, $\pi$ electrons need a positive charge for the interaction to occur. Identification of $\pi-\pi$ interactions firstly look for atoms that are commonly involved:

- sp2 carbons with hydrogen anywhere,

- $\mathrm{C} / \mathrm{N} / \mathrm{O} / \mathrm{S}$ atoms in planar rings.

For Carbons in planar rings are additionally check takes place. It is taken for searching for bond electron-withdrawing (electronegative) atoms that invert the charge distribution.

After that, YASARA assigns simple default charges to all those atoms (+-0.15 to $\mathrm{H}-\mathrm{C}$ and +-0.3 to $\mathrm{H}-\mathrm{N} / \mathrm{N} / \mathrm{O} / \mathrm{S}$ in rings). When this is completed, another calculation is involved, this time to calculate the electrostatic interaction energies. In the final, it maps them to an interaction strength in the range 0 (none) to 1 (optimal).

The third iterations are cation $-\pi$. Those interactions are between $\pi$ electrons, and cations are considered in the same way as the $\pi-\pi$ interactions were. Here the interaction partners are required to be cation. In this case, the charge is taken as the formal charge.

For each experimental setting simulation were performed for 5 distinct realizations in order to account for an impact of random factors on simulations and collect statistics. 


\section{Results}

Major results of simulations are presented in this section. Numbers of hydrogen bonds and HP contacts are presented in Figure 4. Interestingly at the initial part of simulations number of HP contacts rises, next it stays constant. For other temperature as well as cation $-\pi$ and $\pi-\pi$ interactions see Appendix A. Results seems to be temperature independent.

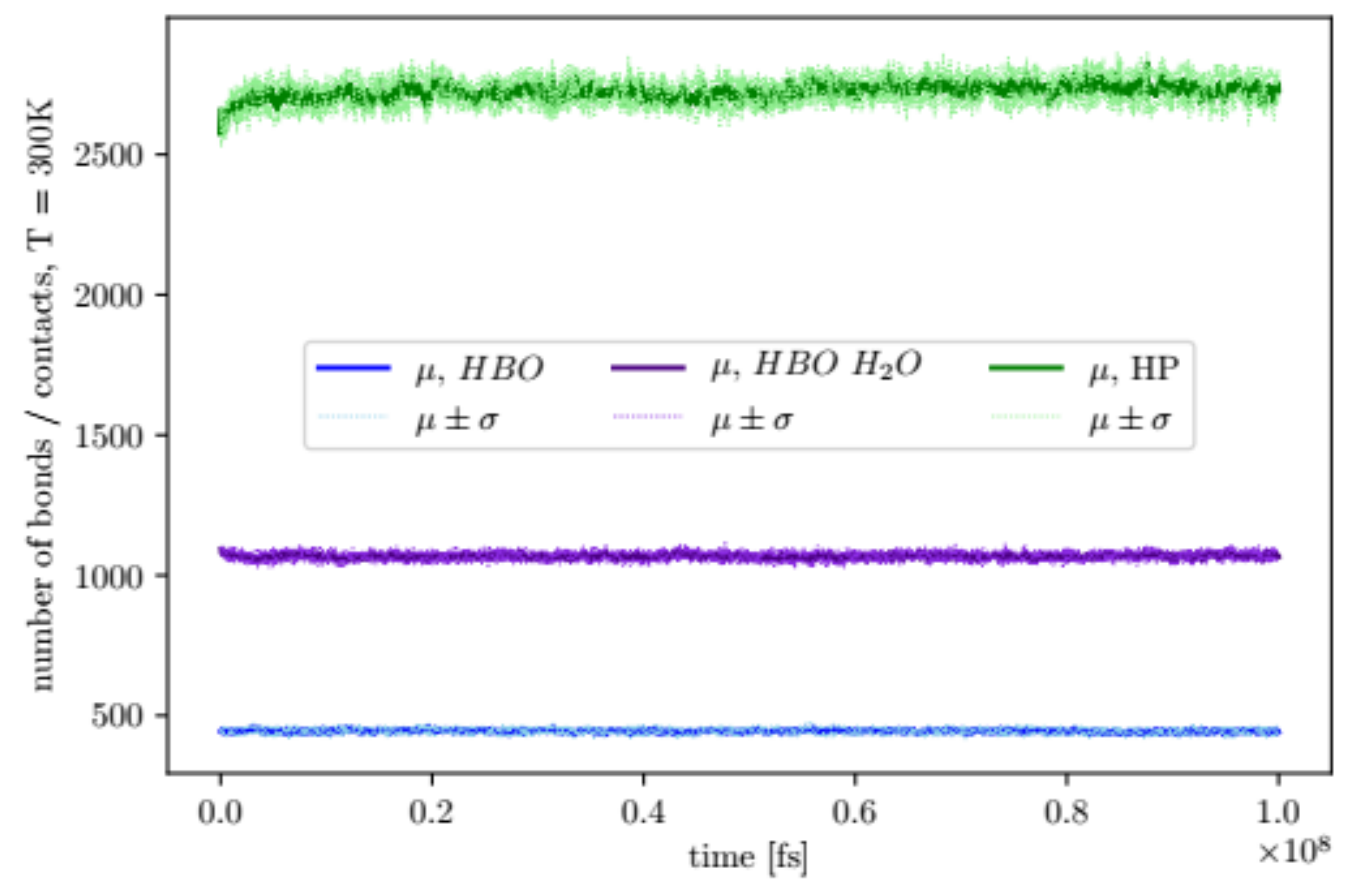

Figure 4. Number of hydrogen bonds and HP contacts, for an exemplary temperature of 300K. Mean and standard deviation were calculated over 5 realizations of experiments.

In Figure 5 we present aggregate histograms of interactions and contacts lengths. Aggregations were preformed over simulation time moments and realizations. Observe the negative skewness and some sort of the cut-off at $5.0^{*} 10^{-10} \mathrm{~m}$ for HP contacts. Histograms of hydrogen bonds lengths appear to be much more symmetric. For skewness on particular simulation moments and various temperatures see Appendix B.
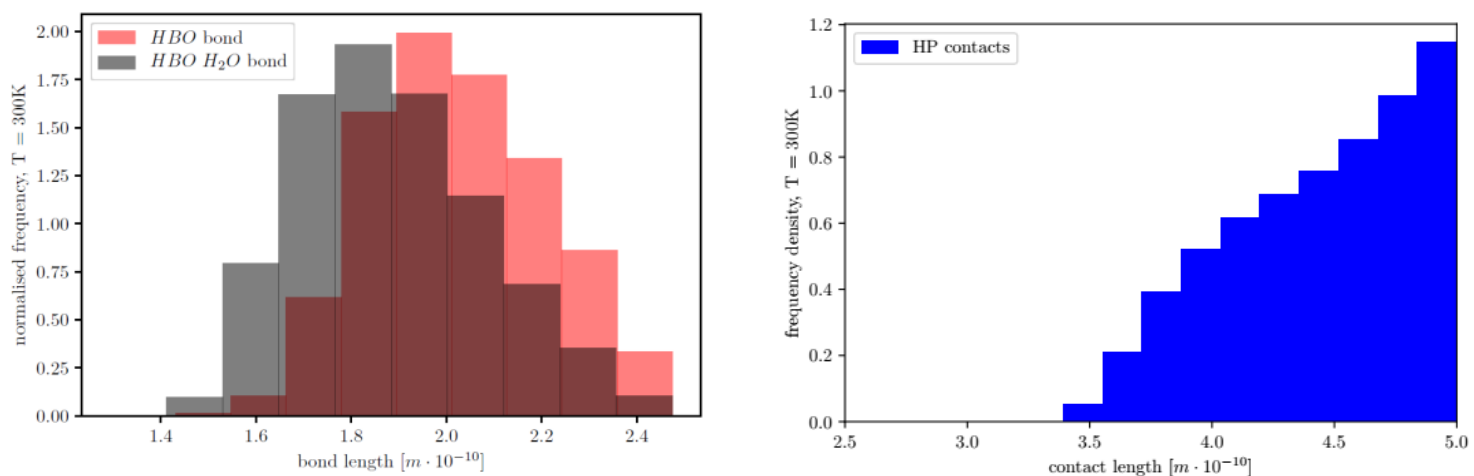

Figure 5. Aggregate histograms of hydrogen bonds interactions lengths (left panel), and HP contacts lengths (right panel), exemplary temperature $\mathrm{T}=300 \mathrm{~K}$. Observe different mode length and skewness. Results for other temperatures are similar, see Appendix B. 


\subsection{Spectrum analysis of the bond energy.}

Following [15], we compute a total energy of each bond interaction and each simulation time and analyze its power spectrum. Exemplary scaling is presented in Figure 6. Scaling exponents (including standard errors from realizations) are presented in Figure 7 for all data. Exponents of hydrogen bonds do not overlap. Although dynamics of these bonds seams to differ, yet all of them show up the characteristic of white noise.

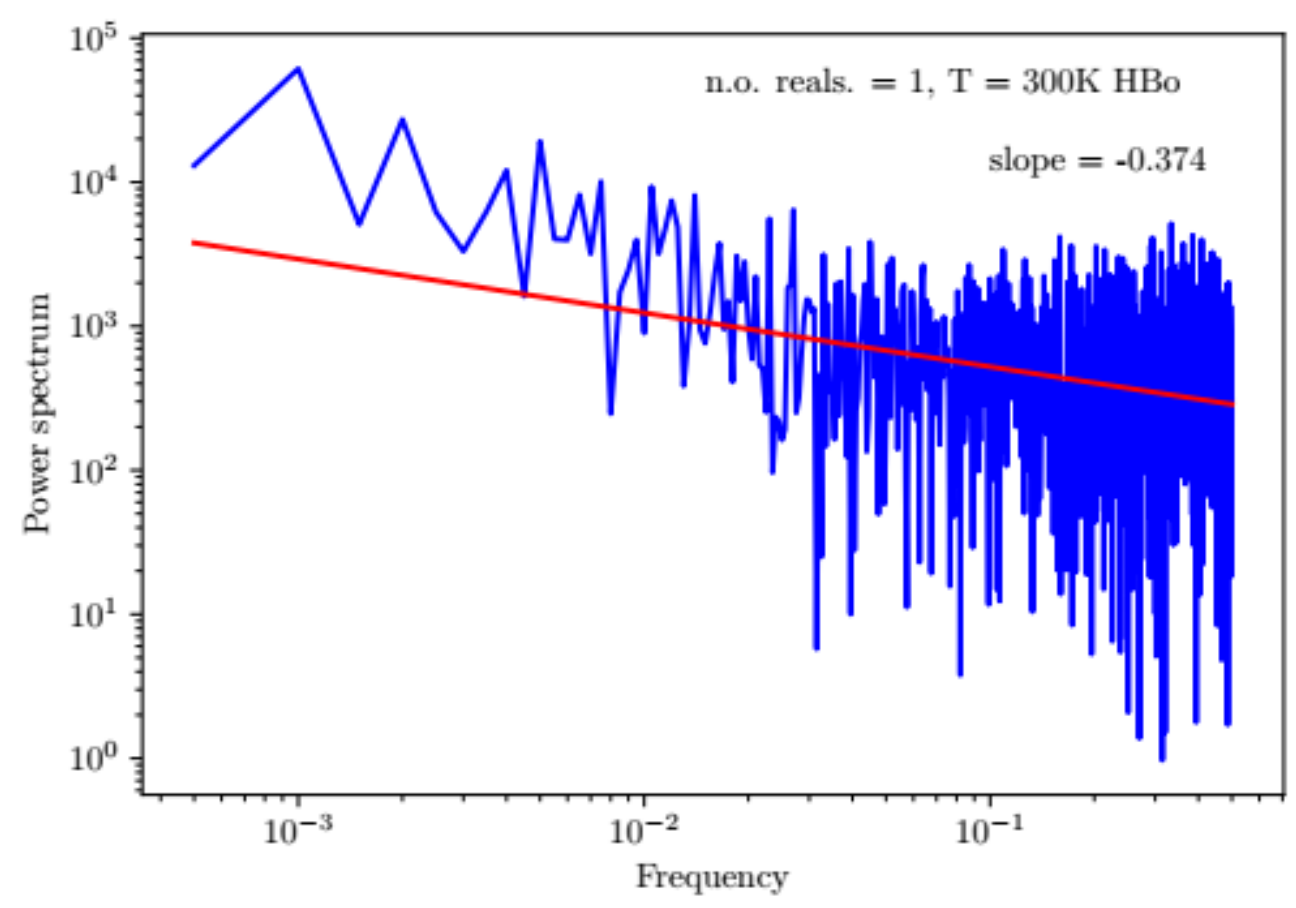

Figure 6. Exemplary scaling of the power spectrum of $\mathrm{HBO}$ bonds energy for given temperature and realization.
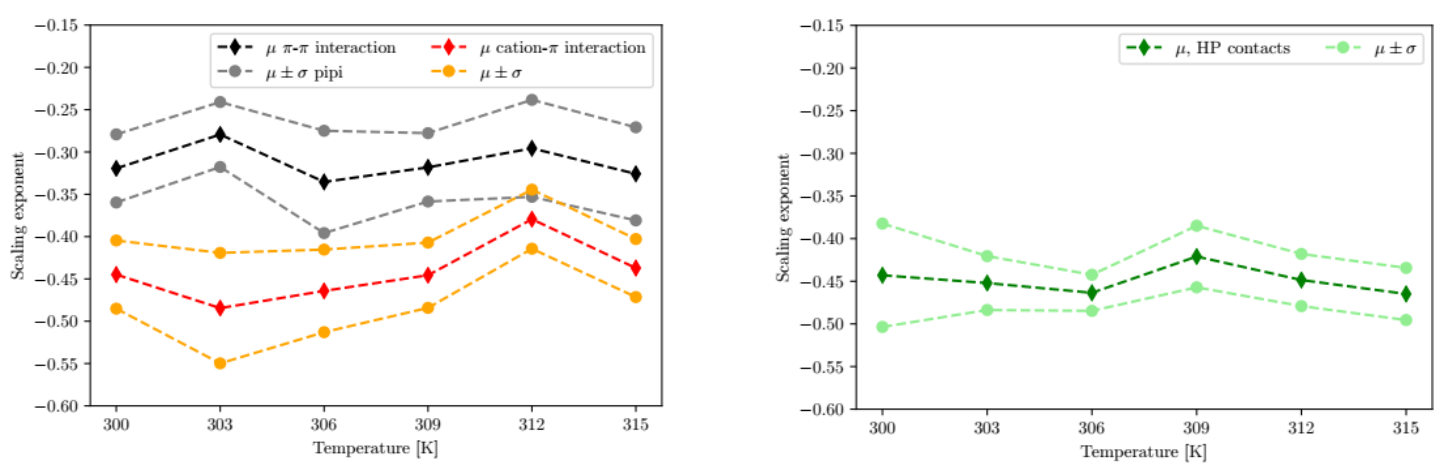


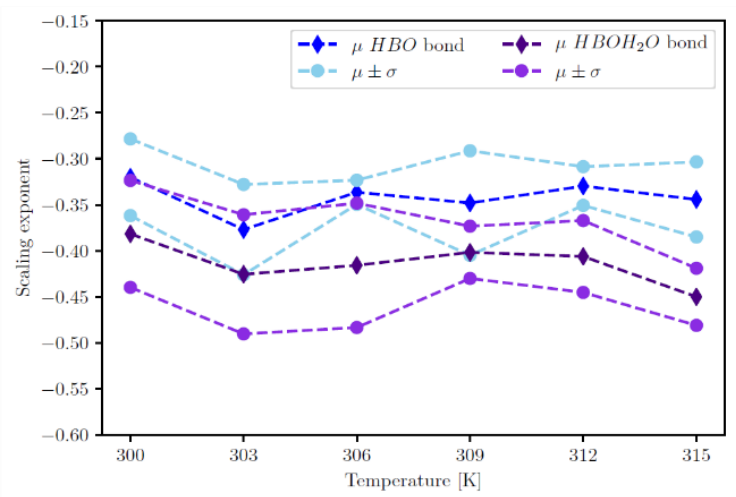

Figure 7. Exponents of power spectra for all types of interactions / contacts / bonds and temperatures in range $300 \mathrm{~K}-315 \mathrm{~K}$. All exponents are in ranges -0.2 to -0.55 . Exponent differ meaningfully between distinct hydrogen bonds and distinct interactions. NP contacts and cation- $\pi$ interaction seem to have similar scaling exponents.

Scaling exponents of power spectra are in range -0.2 to -0.55 what is a more narrow range than this recorded in [17]. Scaling exponent differ meaningfully between intramolecular and solvent-solute HBs what suggest a different dynamics between these bonds. This was approved by distinctly different decay dynamics and these types of bonds. Next cation $-\pi$ and $\pi-\pi$ interactions have significantly different scaling exponent, but cation- $\pi$ interaction and HP contacts seem to have a similar scaling exponent.

\subsection{Analysis of particular amino-acids interactions..}

There are 20 amino-acids building the albumin. Following [17], in this subsection we analyze between which amino-acids bonds / interactions or contacts are formed. In the case of the $\pi-\pi$, cation $-\pi$, H-bonds and HP contacts the connection is formed between two amino-acids, for interaction map see Figure 8 and 9. In case of the albumin- $\mathrm{H}_{2} \mathrm{O} \quad \mathrm{HB}$ it is formed between the single amino acid and the $\mathrm{H}_{2} \mathrm{O}$ particle, see histogram in Figure 10. It seems that Lysine (LYS) and Glutamine (GLU) plays an important role in both types of hydrogen bonds. In case of NP contacts it seems that Phenylalanine (PHE) and (LEU) plays a major role.

For H-bonds most are formed between Lysine (LYS) and Glutamine(GLU), and bond between GLU and Aspartic acid (ASP). Interactions between LYS -ASP, LYSLEU, LYS-ALA and ALA-LEU also have the nature of hydrogen bonds.

The same amino acids, which form more HB between themselves, also form the most $\mathrm{HB} \mathrm{H} \mathrm{H}_{2} \mathrm{O}$ interactions: GLU, ASP and LYS (Figure 10). It is noteworthy that GLU and ASP are proton acceptors, whereas LYS is a donor of proton.

Referring to particular amino-acids connections, it appears that most $\pi-\pi$ interactions are between Phenylalanine (PHE) and itself, most of cation $-\pi$ are between Tyrosine (TYR) and Arginine (ARG). This is easy to predict as only these amino acids contain aromatic rings (i.e. de-localized pi electrons that can interact with the pi electrons of other rings or a positively charged arginine group $=\mathrm{NH}_{2}{ }^{+}$). 

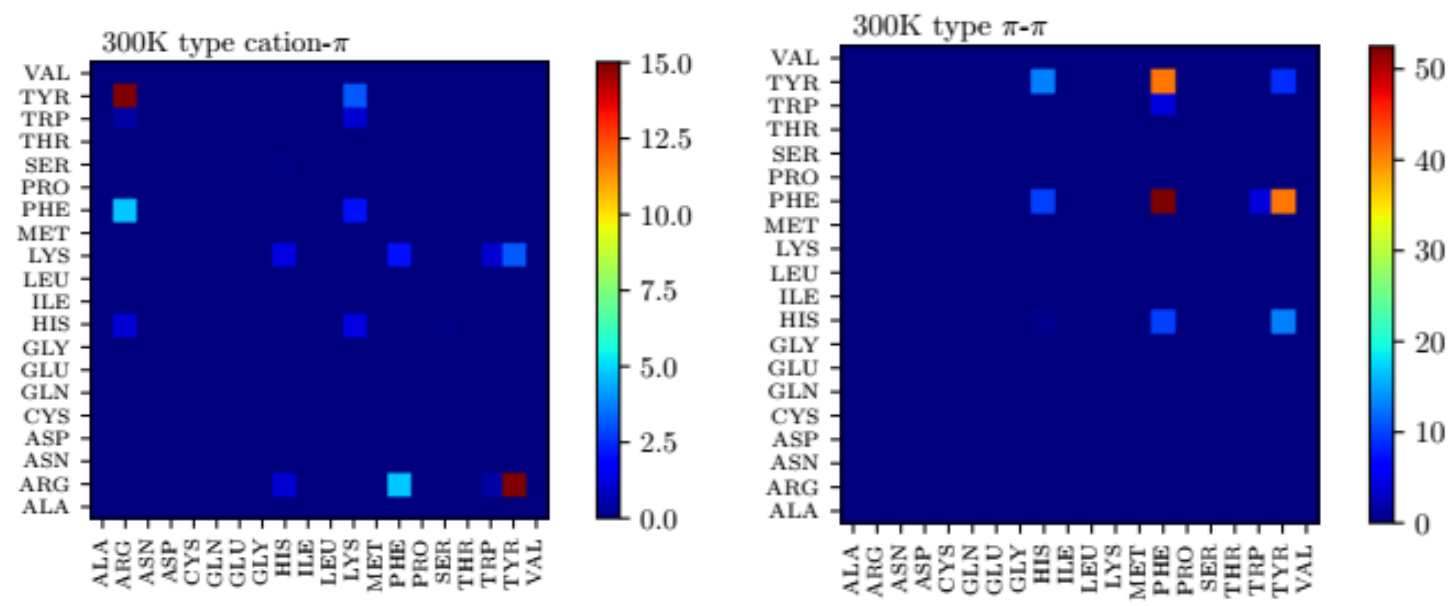

Figure 8. Maps of $\pi-\pi$, cation $-\pi$ interactions between particular amino-acids. For $\pi-\pi$ most interactions are between Phenylalanine (PHE) and Phenylalanine (PHE) and between Tyrosine (TYR) and Phenylalanine (PHE), for cation $-\pi$ between Tyrosine (TYR) and Arginine (ARG), $\mathrm{T}=300 \mathrm{~K}$.
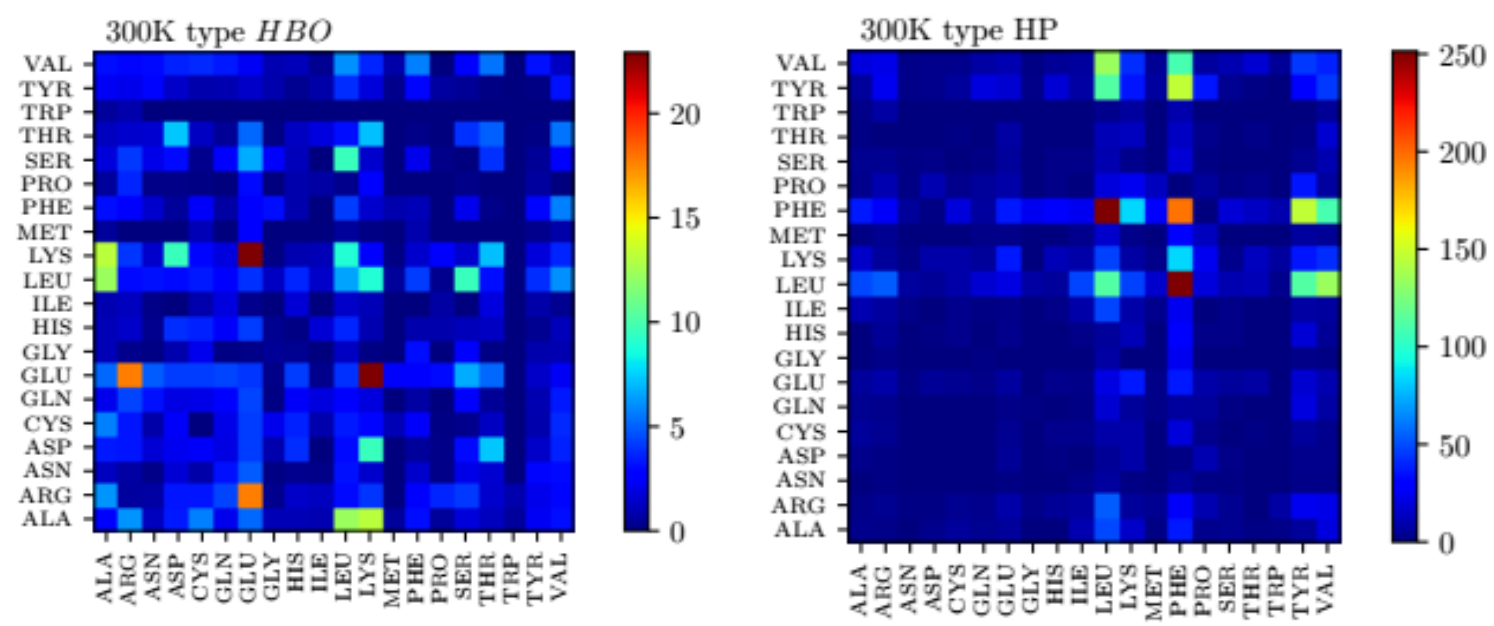

Figure 9. Maps of HBO hydrogen bonds (left panel) and HP contacts (right panel) between particular amino-acids. For HBO bonds there are much more combinations than in the case of contacts, here most bonds are formed with Lysine (LYS). In case of NP contacts Phenylalanine (PHE) and Leucine (LEU) plays a major role, $\mathrm{T}=300 \mathrm{~K}$.

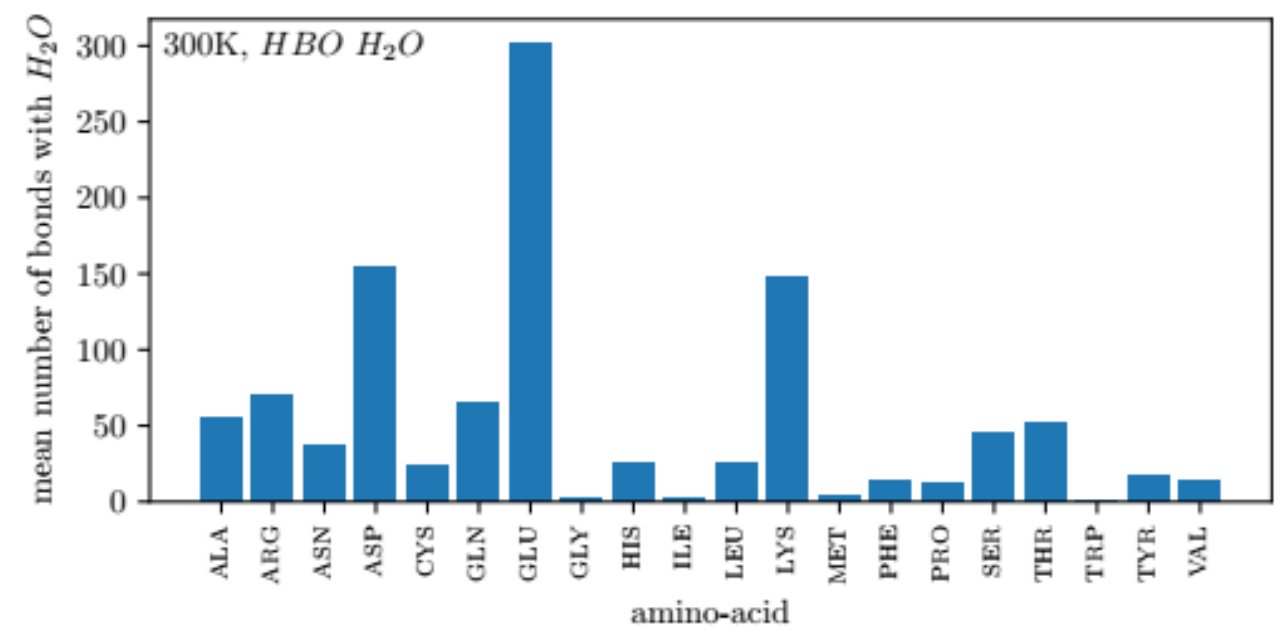

Figure 10. Histograms of $\mathrm{HB} \mathrm{H}_{2} \mathrm{O}$ contacts with water particles, it appears that Glutamine(GLU) plays an important role, $\mathrm{T}=300 \mathrm{~K}$. 


\subsection{Stability (living time) of bonds interactions and contacts.}

Following analysis in [18] we present decay curves of particular bonds / contacts and interactions. At first particular types of bond / contact or interaction is recognized after one simulation step (to avoid any inconsistency with initial conditions). Next we compute how much of these survived after subsequent simulation steps and present its fraction in Figure 11. Observe a large difference in decay rate between two types of hydrogen bonds.

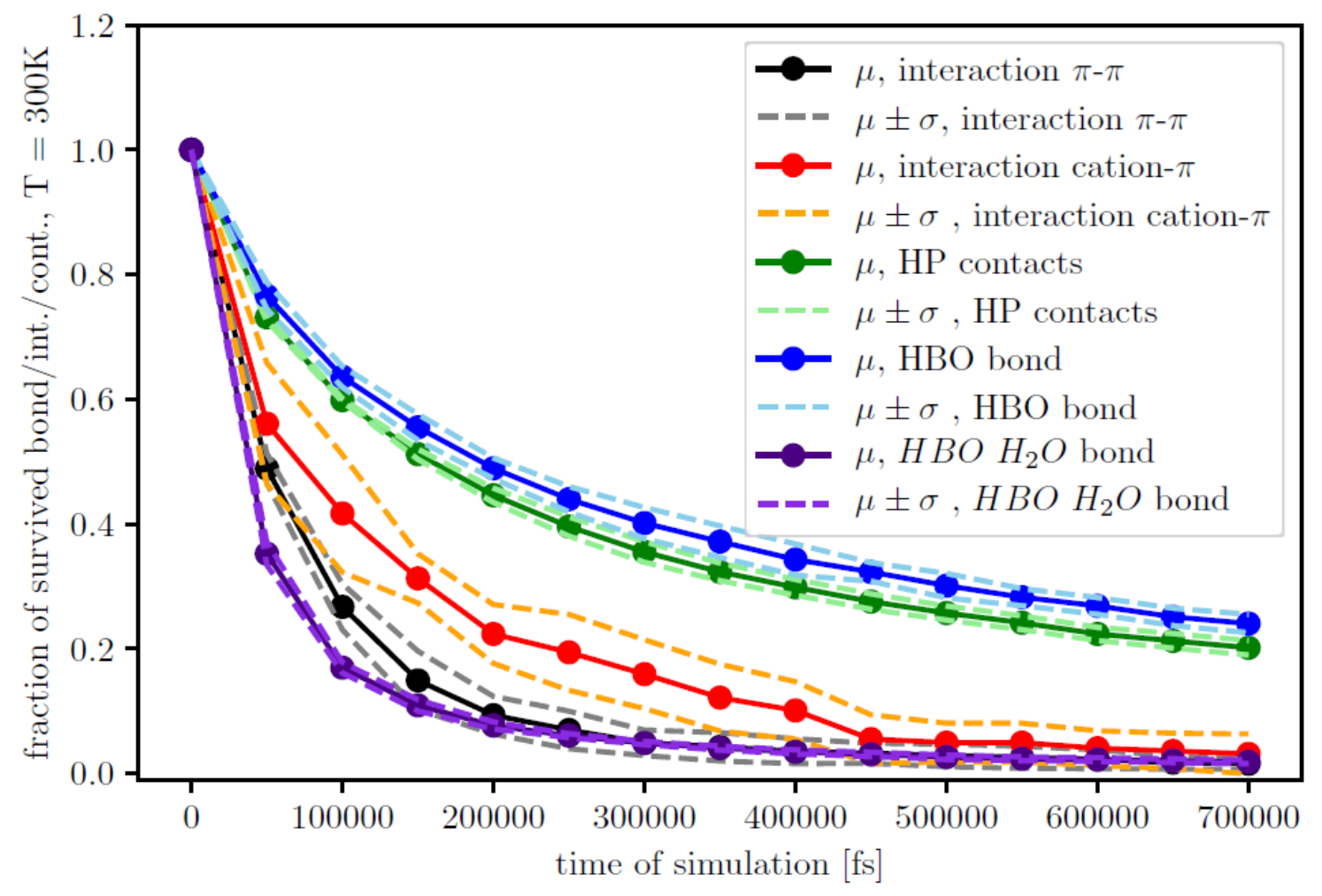

Figure 11. Decay curve for various types of bonds interactions and contacts, statistics were taken over realizations. Observe that $\mathrm{HBO} \mathrm{H}_{2} \mathrm{O}$ bonds as well as $\pi-\pi$ interactions decay in fastest rate, while $\mathrm{HP}$ contacts and $\mathrm{HBO}$ bonds in slowest rate, $\mathrm{T}=300 \mathrm{~K}$.

Survival histograms of HP contacts and hydrogen bonds between chosen amino-acids and all amino-acids (or water particles in $\mathrm{HBO} \mathrm{H}_{2} \mathrm{O}$ ) are presented in Figures 12 - 14. For HP constants most stable in Tyrosine (TYR), for HBO bonds Leucine (LEU) while for $\mathrm{HB}_{2} \mathrm{O}$ - Arginine (ARG). 


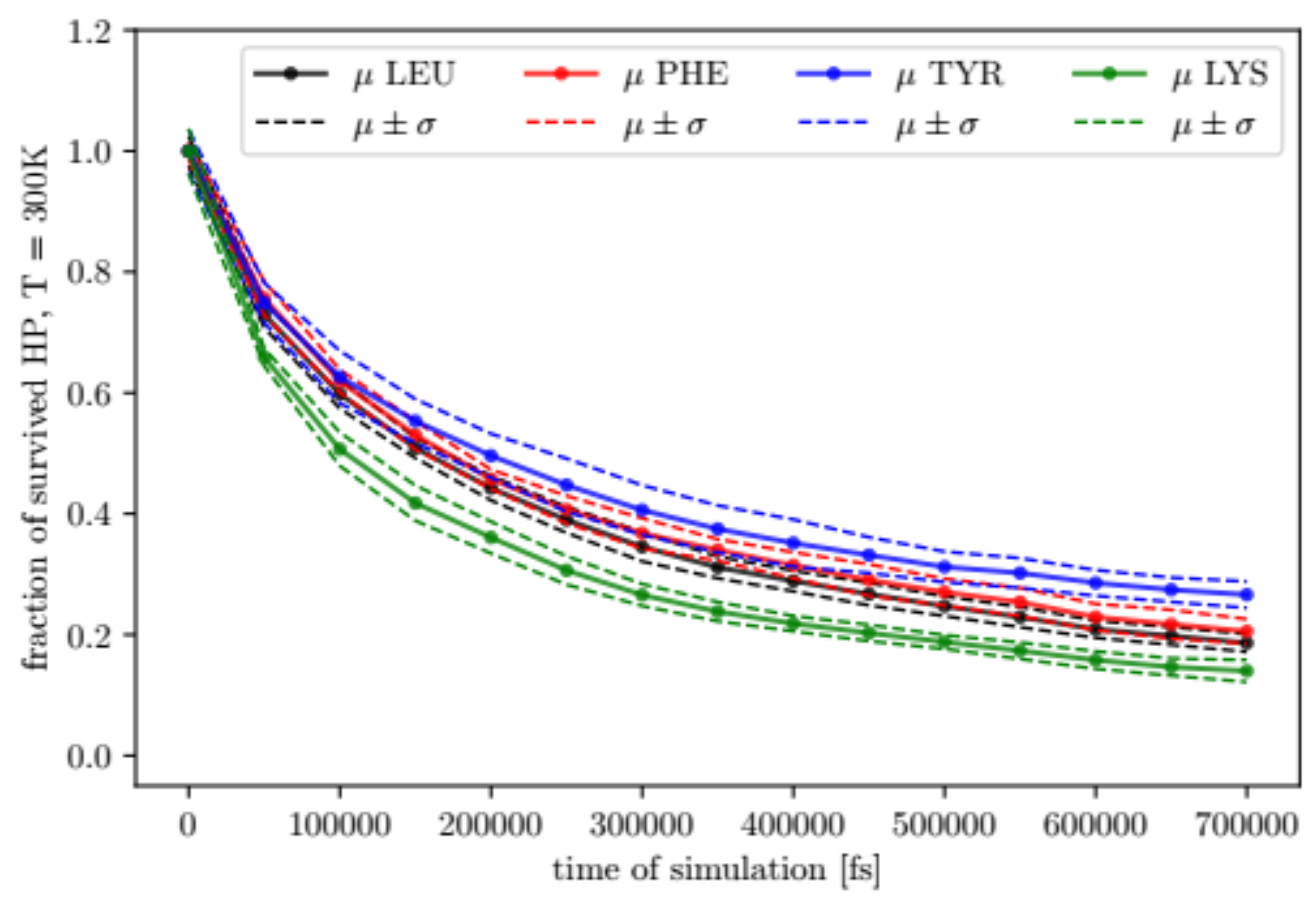

Figure 12. Decay plot of HP contacts between chosen amino-acid and all amino-acids, $\mathrm{T}=300 \mathrm{~K}$. Tyrosine (TYR) produces most stable HP contacts.

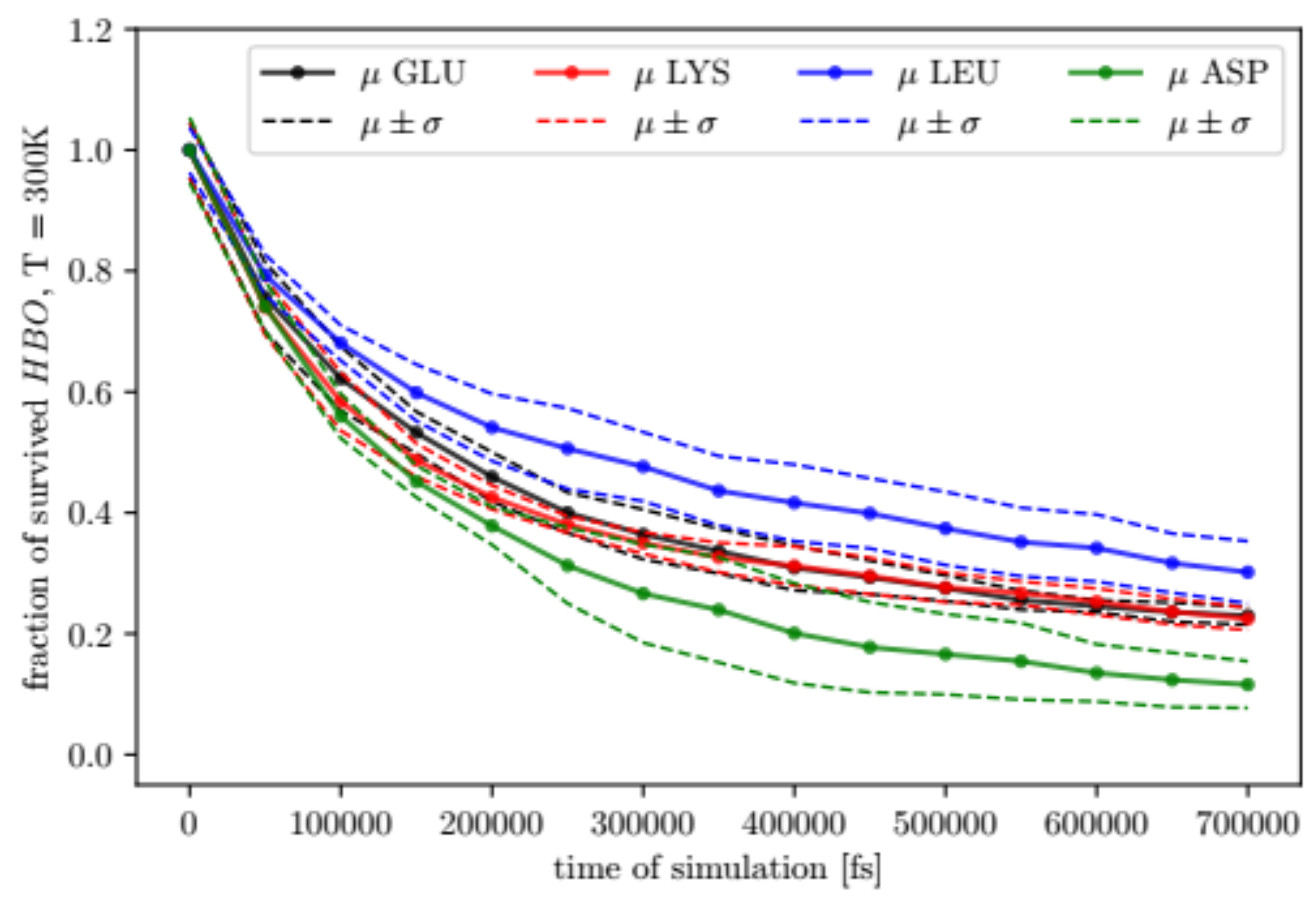

Figure 13. Decay plot of HBO bonds between chosen amino-acid and all amino-acids, $\mathrm{T}=300 \mathrm{~K}$. Leucine (LEU) produces most stable hydrogen bond. 


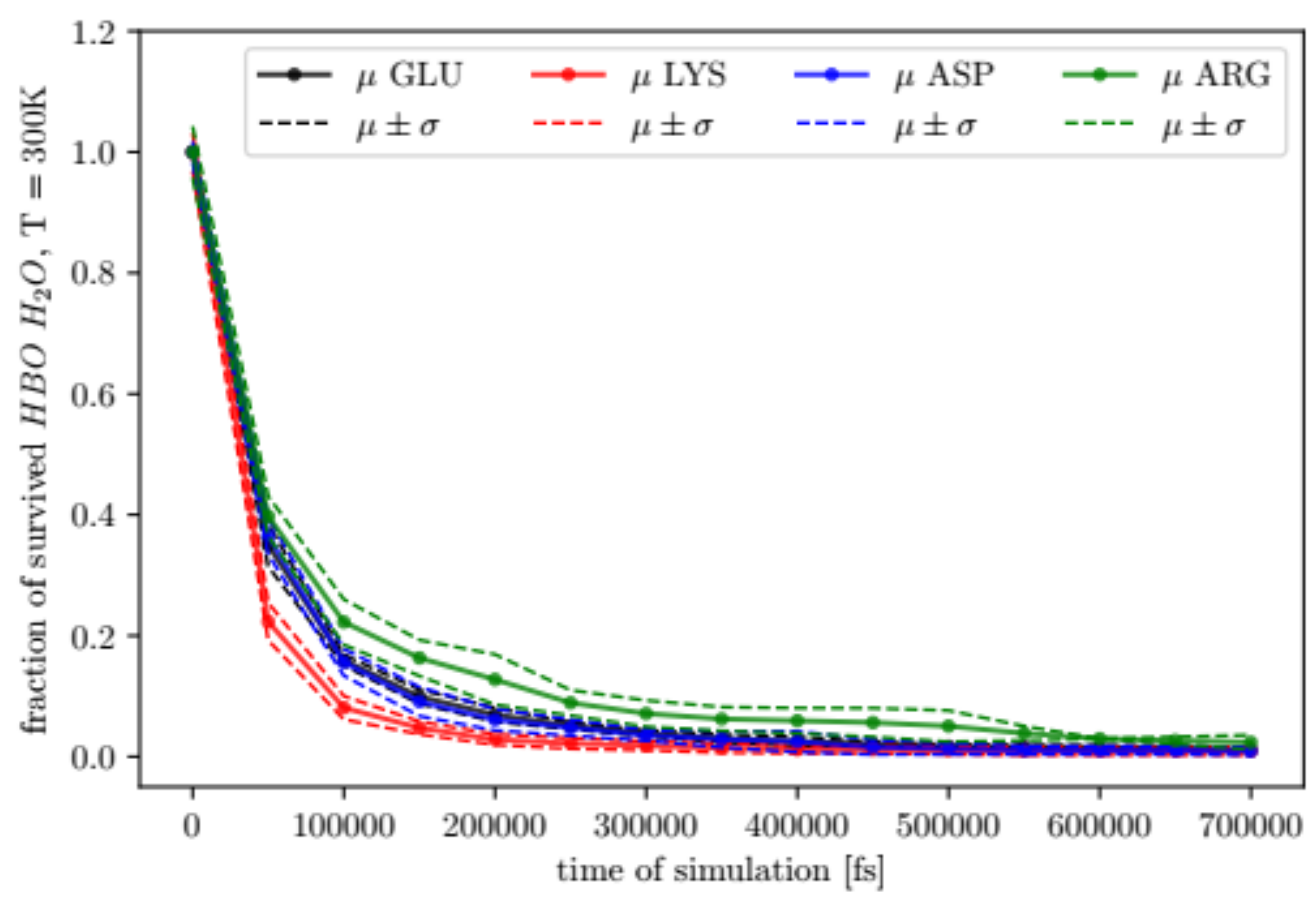

Figure 14. Decay plot of $\mathrm{HBO} \mathrm{H}_{2} \mathrm{O}$ bonds between chosen amino-acid and water particles, $\mathrm{T}=300 \mathrm{~K}$. Arginine (ARG) produces most stable hydrogen bond with water particles.
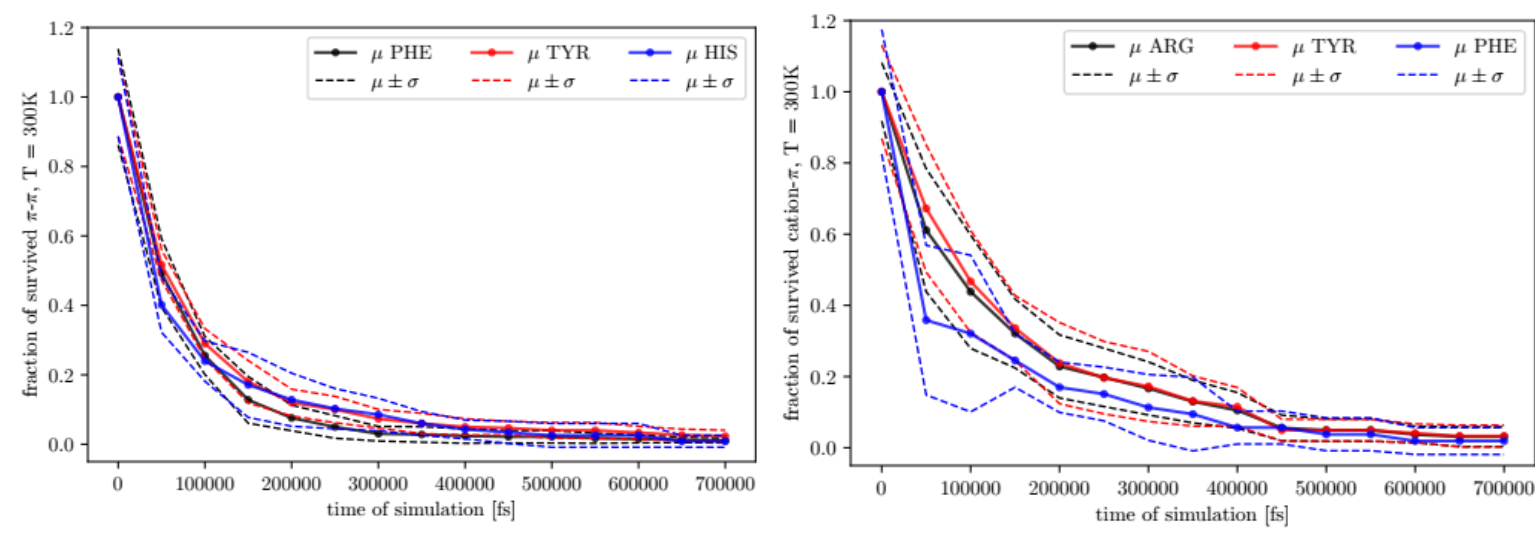

Figure 15. Decay plot of $\pi-\pi$, cation $-\pi$ interactions between chosen amino-acid and all aminoacids, $\mathrm{T}=300 \mathrm{~K}$.

Finally survival histograms of of $\pi-\pi$ and cation $-\pi$ interactions between chosen amino-acids and all amino-acids are presented in Figure 15.

\section{Conclusions}

Presented results show in the molecular detail details of a selected protein in its native, folded state. Properties of all amino acid from the albumin molecule reflects nature of their interactions with other proteins and water. Charged AA, such as GLU, LYS, ASP form mainly hydrogen bonds, see Figure 8. On the other hand hydrophobic AA: PHE, LEU, TYR and VAL create most of hydrophobic contacts as shown in the Figure 9 . It is also worth to mention that LEU show up high binding for both HB, HP and low with water. This indicates its position in the protein as it 
is buried in the core of albumin. Interestingly LEU forms most stable HB with water, which also reflect above mention position, as water molecules can be trapped. LYS, GLU, ASP and ARG as charged AA can be found at the surface, thus intramolecular bonding can be replace with solvent HB. ARG form less HB with water than other charged AA but it tend to last longer as presented in Figure 14.

The studies performed by us, in which sampling took place every 50 ps, give preliminary results, which are needed to study the stability of proteins, modeling of their reactions and intramolecular and intermolecular interactions.

Author Contributions: conceptualization, R.D., K.D. and D.B.; methodology, R.D., K.D. and D.B.; software, K.D..; validation, R.D., D.B., A.M.; formal analysis, K.D., R.D., D.B.; investigation, K.D., R.D., D.B.; resources, K.D., R.D., D.B.; data curation, K.D., R.D., D.B.; writing-original draft preparation, K.D., R.D., D.B., A.M.; writing-review and editing, A.M., J.M., M.S.; visualization. R.D., D.B., J.M., M.S., A.M.; supervision, K.D, R.D..; project administration, A.M., J.M., M.S.

Funding: This research received no external funding.

Acknowledgments: The authors would like to thank Dr. Piotr Bełdowski for his great help in analyzing of the data and preparing the manuscript. We would like to thank the Mechanical Engineering Department, UTP University of Science and Technology in Bydgoszcz, for financial support.

Conflicts of Interest: The authors declare no conflict of interest.

\section{Appendix A}

In Appendix A we present numbers of interactions, contacts and bonds for various temperatures. For hydrogen bonds and HP contacts see Figure $1 \mathrm{~A}$, for $\pi-\pi$ and cation- $\pi$ interactions see Figure $2 \mathrm{~A}$. Observe that for the first stage of simulation time, some of cation $-\pi$ interactions are replaced by $\pi-\pi$ ones and this phenomenon is roughly temperature independent (at least in the temperature range of $300 \mathrm{~K}-315 \mathrm{~K})$.
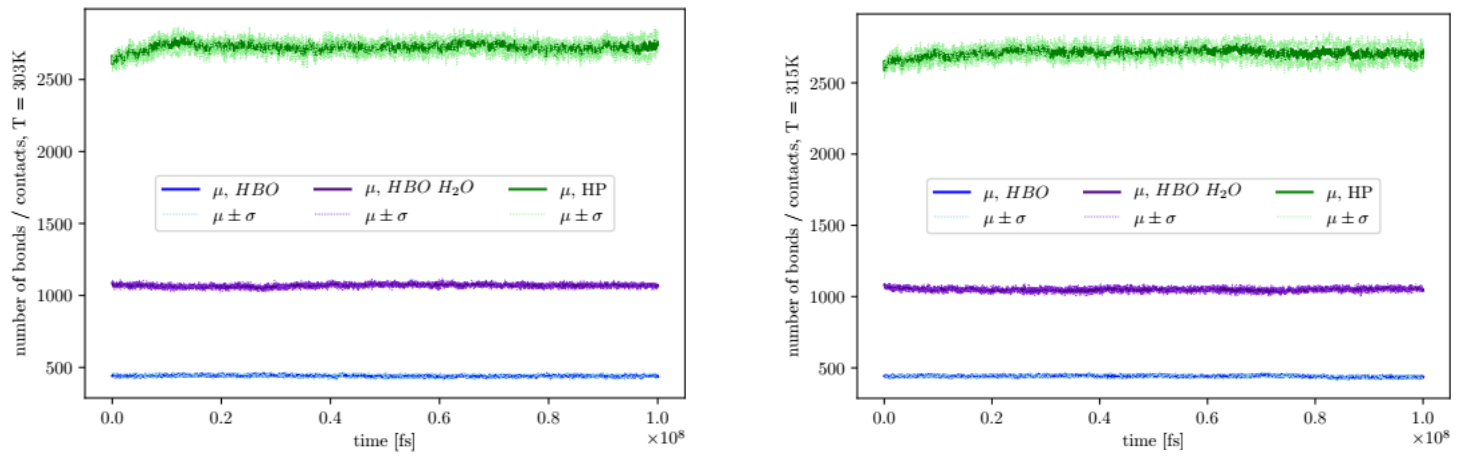

Figure 1A. Number of hydrogen bonds, and HP contacts for $\mathrm{T}=303 \mathrm{~K}$ and $315 \mathrm{~K}$, mention that $\mathrm{T}=$ $300 \mathrm{~K}$ case is in the main body text. One can see at the first stage of simulations number of HP contacts rises, next it is roughly constant. This rise seems to be more smooth as a temperature rises. 

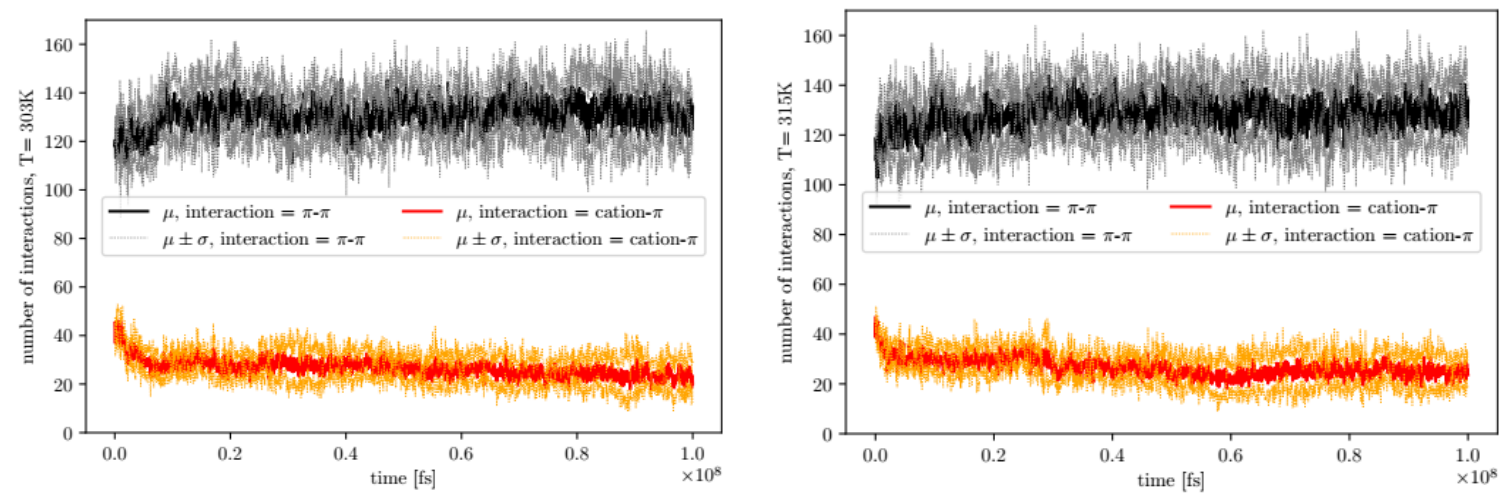

Figure 2A. Number of interactions for various temperatures: $303 \mathrm{~K}$ and $315 \mathrm{~K}$, mention that $300 \mathrm{~K}$ is similar to $303 \mathrm{~K}$. Observe that at the first stage some of simulation some $\pi-\pi$ contacts are replace by cation $-\pi$.

\section{Appendix B}

In this appendix we present aggregate histograms of bonds lengths for various temperatures. Aggregation realizations and stimulation time moments. Histograms of cation $-\pi$ and $\pi-\pi$ interactions and HP contacts are stair like and negative skewed, see Figures $2 \mathrm{~B}$ and $3 \mathrm{~B}$. This skewness appears to be simulation step independent and temperature independent, see Figure $4 \mathrm{~B}$. This is in contrary to hydrogen bond length histograms, see Figure 1B.
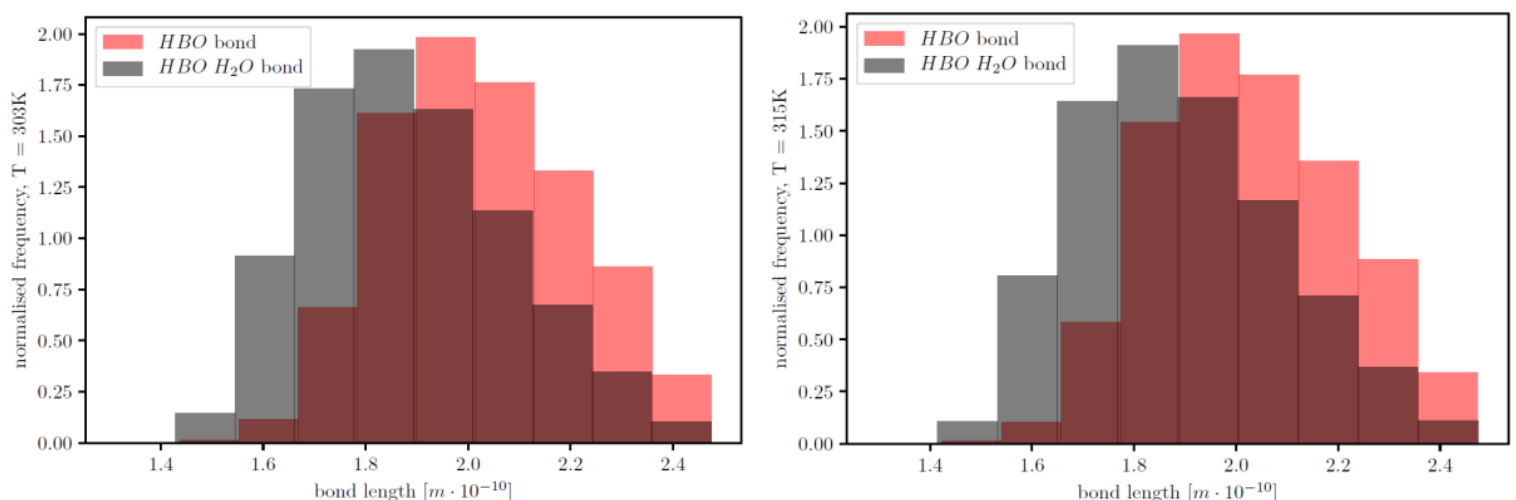

Figure 1B. Aggregate histogram of hydrogen bonds lengths, $T=303 \mathrm{~K}$ and $\mathrm{T}=315 \mathrm{~K}$, mention that $\mathrm{T}=300 \mathrm{~K}$ is in the main body text.
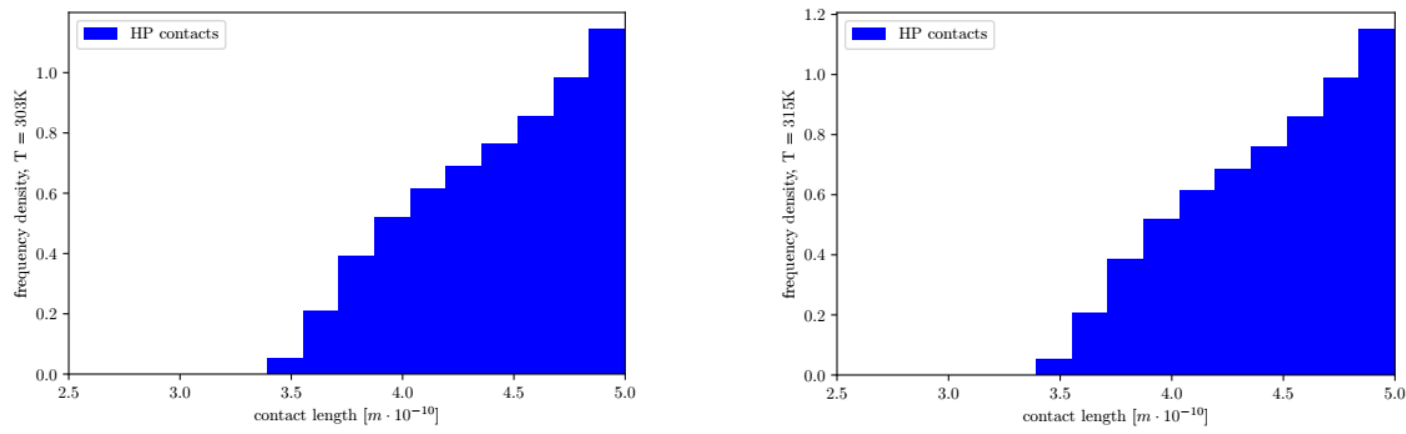

Figure 2B. Aggregate histogram of $\mathrm{HP}$ contacts length, $\mathrm{T}=303 \mathrm{~K}$ and $\mathrm{T}=315 \mathrm{~K}$, mention that $\mathrm{T}=300 \mathrm{~K}$ is in the main body text. 

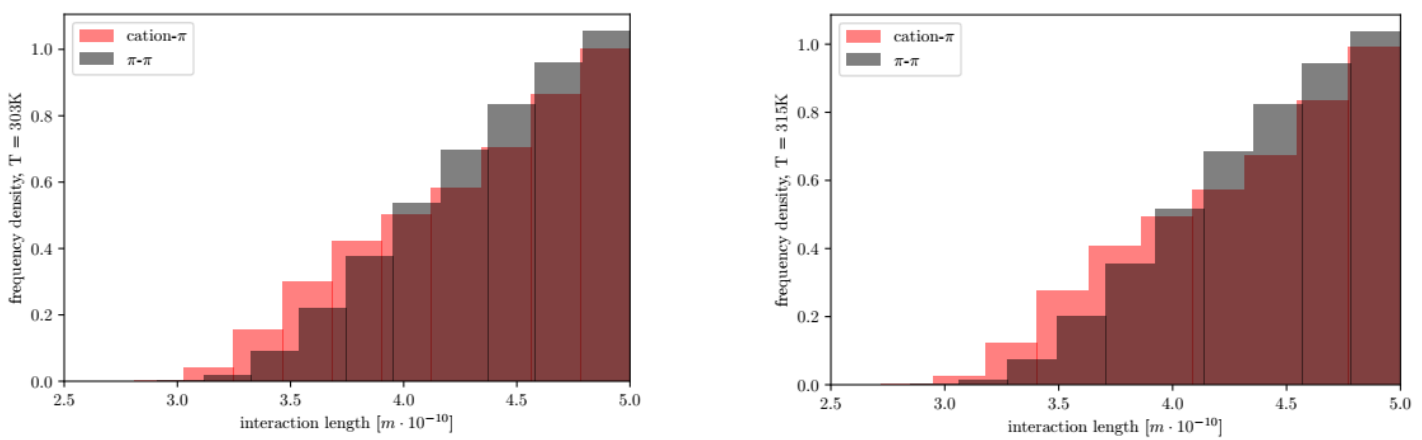

Figure 3B. Aggregate histogram of cation $-\pi$ and $\pi-\pi$ interactions, $T=303 \mathrm{~K}$ and $\mathrm{T}=315 \mathrm{~K}$, mention that $\mathrm{T}=300 \mathrm{~K}$ outcome is similar to $\mathrm{T}=303 \mathrm{~K}$.
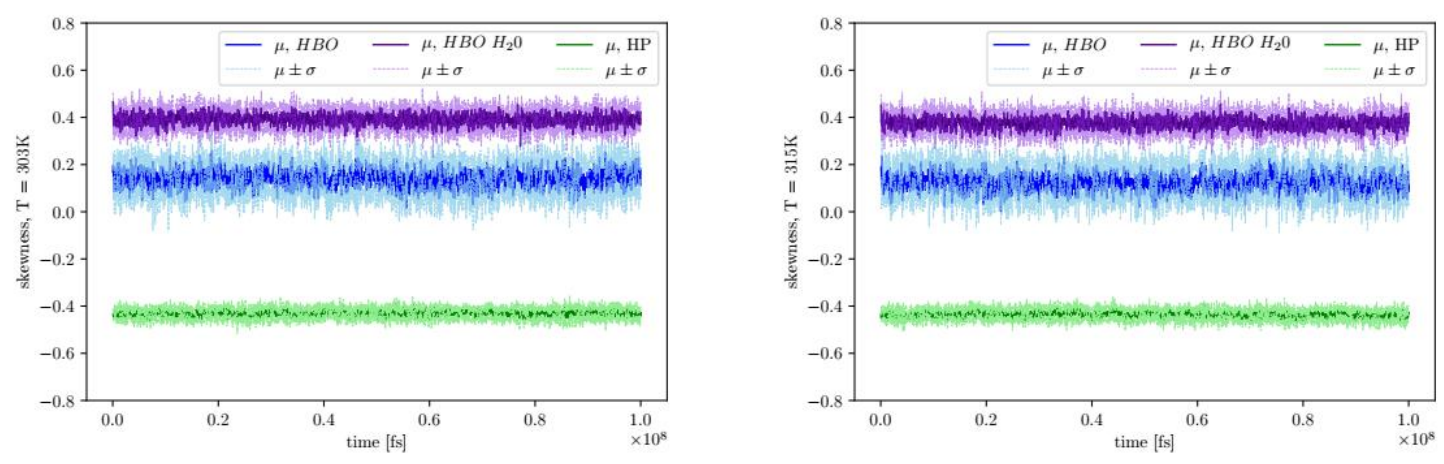

Figure 4B. Skewness for histograms for particular simulation time steps, HP contacts and hydrogen bonds, $\mathrm{T}=303 \mathrm{~K}$ and $\mathrm{T}=315 \mathrm{~K}$. Observe that skewness is time independent. For hydrogen bonds we have positive skewness while for HP we have negative one.

\section{References}

1. Peters T. All About Albumin: Biochemistry, Genetics, and Medical Applications. San Diego, CA: Academic Press, 1996.

2. Bennike, T.; Ayturk, U.; Haslauer, C.M.; Froehlich, J.W.; Proffen, B.L.; Barnaby, O.; Birkelund, S.; Murray, M.M.; Warman, M.L.; Stensballe, A.; Steen, H. A Normative Study of the Synovial Fluid Proteome from Healthy Porcine Knee Joints. J. Proteome Res. 2014, 13, 4377-4387. DOI: 10.1021/pr500587x

3. Bhattacharya, A.A.; Curry, S.; Franks, N.P. Binding of the General Anesthetics Propofol and Halothane to Human Serum Albumin. J. Biol. Chem. 2000, 275, 38731-38738. DOI 10.1074/jbc.M005460200

4. Evans, T.W. Review article: albumin as a drug-biological effects of albumin unrelated to oncotic pressure. Aliment. Pharmacol. Ther. 2002, 16, 6-11. DOI: 10.1046/j.1365-2036.2002.00190.x

5. Quinlan, G.J.; Martin, G.S.; Evans, T.W. Albumin: Biochemical Properties and Therapeutic Potential. Hepatology 2005, 41, 1211-1219. DOI 10.1002/hep.20720

6. Subha Mahadevi, A.; Narahari Sastry, G. Cooperativity in Noncovalent Interactions. Chem. Rev. 2016, 116, 2775-2825. DOI: $10.1021 / \mathrm{cr} 500344 \mathrm{e}$ 
7. Cerny, J.; Hobza, P. Non-covalent interactions in biomacromolecules. Phys. Chem. Chem. Phys. 2007, 9, 52915303. DOI: $10.1039 / \mathrm{b} 704781 \mathrm{a}$

8. Dedinaite, A.; Wieland, D.C.F.; Bełdowski, P.; Claesson, P.M. Biolubrication synergy: HyaluronanPhospholipid interactionsat interfaces. Adv. Colloid Interfac. 2019, 274, 102050. DOI: 10.1016/j.cis.2019.102050

9. Bełdowski, P.; Yuvan, S.; Dedinaite, A.; Claesson, P.M.; Pöschel, T. Interactions of a short hyaluronan chain with a phospholipid membrane. Colloid Surface. B 2019, 110539. DOI: 10.1016/j.colsurfb.2019.110539

10. Bełdowski, P.; Weber, P.; Dedinaite, A.; Claesson, P.M.; Gadomski, A. Physical crosslinking of hyaluronic acid in the presence of phospholipids in an aqueous nano-environment. Soft Matter 2018, 14, 8997-9004. DOI: $10.1039 / \mathrm{c} 8 \mathrm{sm} 01388 \mathrm{~h}$

11. Arunan, E.; Desijaju, G.R.; Klein, R.A.; Sadlej, J.; Scheiner, S.; Alkorta, I.;Clary, D.C.; Crabtree, R.H.; Dannenberg, J.J.; Hobza, P.; Kjaergaard, H.G.; Legon, A.C.; Mennucci, B.; Nesbitt, D.J. Definition of the hydrogen bond (IUPAC Recommendations 2011). Pure Appl. Chem. 2011, 83, 1637-1641. DOI:10.1351/PACREC-10-01-02

12. Parthasarathi, R.; Subramanian, V. Characterization of hydrogen bonding: from van der Waals interaction to covalency. In Hydrogen Bonding-New Insights, Grabowski, S.J.; Springer, Dordrecht, 2006.

13. Van der Spoel, D.; van Maaren, P.J.; Larsson, P.; Timneanu, N. Thermodynamics of Hydrogen Bonding in Hydrophilic and Hydrophobic Media. J. Phys. Chem. B 2006, 110, 4393-4398. DOI: 10.1021/jp0572535

14. Cerar, J.; Jamnik, A.; Pethes, I.; Temleitner, L.; Pusztai, L.; Tomšič, M. Structural, rheological and dynamic aspects of hydrogen-bonding molecular liquids: Aqueous solutions of hydrotropic tert-butyl alcohol. J. Coll. Inter. Sci. https://doi.org/10.1016/j.jcis.2019.10.094

15. Connoly, M.L. Analytical molecular surface calculation. J. Appl. Cryst. 1983, 16, 548-558. DOI: $10.1107 /$ S0021889883010985

16. Richmond, T.J. Solvent accessible surface area and excluded volume in proteins. Analytical equations for overlapping spheres and implications for the hydrophobic effect. J. Mol. Biol. 1984, 178, 63-89. DOI: 10.1016/0022-2836(84)90231-6

17. Siódmiak J.; Bełdowski P., Hyaluronic Acid Dynamics and its Interaction with Synovial Fluid Components as a Source of the Color Noise. Fluctuation Noise Lett, 2019, 18, 1940013. DOI: 10.1142/S0219477519400133

18. Voloshin, V.P.; Naberukhin Yu.I. Hydrogen bond lifetime distributions in computer-simulated water. J. Struct. Chem. 2009, 50, 78-89. DOI: 0022-4766/09/5001-0078 